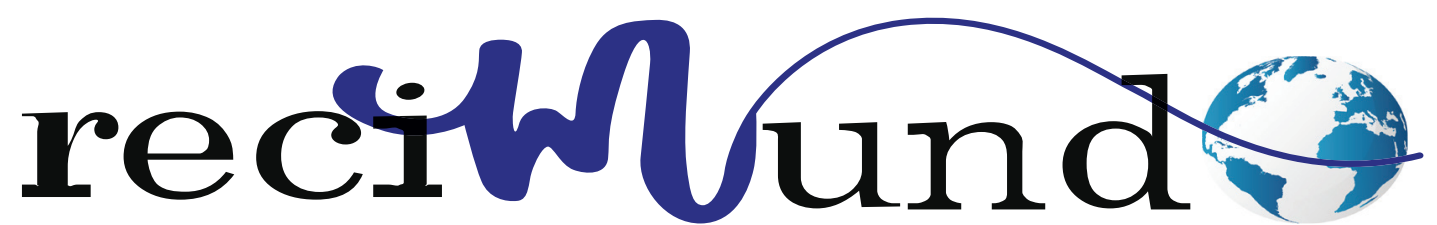

Revista Científica Mundo de la Investigación y el Conocimiento

DOI: 10.26820/recimundo/4.(1).esp.marzo.2020.114-121

URL: http://recimundo.com/index.php/es/article/view/784

EDITORIAL: Saberes del Conocimiento

REVISTA: RECIMUNDO

ISSN: 2588-073X

TIPO DE INVESTIGACIÓN: Artículo de Revisión

CÓdIGO UNESCO: 3201 Ciencias Clínicas; 3201.04 Patología Clínica "sechgacticia. LTDA.
PAGINAS: $114-121$

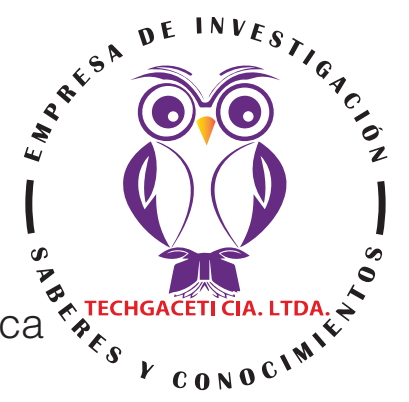

\title{
Diagnóstico en cáncer de pene
}

\section{Diagnosis in penile cancer}

\section{Diagnóstico em câncer de pênis}

Katherine Lizbeth Garcés Erazo; Viviana Angie Quisilema Ron²; Karina Elizabeth Pacheco Romero;

Darwin Ramiro Bungacho Moreno 4

\section{RECIBIDO: 20/11/2019 ACEPTADO: 29/01/2020 PUBLICADO: 05/03/2020}

1. Médico; Investigadora Independiente; Quito, Ecuador; lizbethge123512@gmail.com; (DD https://orcid.org/00000001-8027-8384

2. Médico; Investigadora Independiente; Quito, Ecuador; angiee_30918@hotmail.com; (D) https://orcid.org/00000002-1034-3950

3. Médico; Investigadora Independiente; Quito, Ecuador; kary2102_e@hotmail.com; (D) https://orcid.org/00000001-7115-3942

4. Médico; Investigador Independiente; Quito, Ecuador; darwinbungacho@hotmail.com; (D) https://orcid. org/0000-0002-9713-8318

CORRESPONDENCIA

Katherine Lizbeth Garcés Erazo

lizbethge123512@gmail.com

Quito, Ecuador

〔 RECIMUNDO; Editorial Saberes del Conocimiento, 2020 


\section{RESUMEN}

El cáncer de pene es una enfermedad que afecta las células del organismo, las cuales se multiplican de manera desordenada en el área del aparato reproductivo masculino; es una de las patologías menos frecuentes en el campo de la urología. El diagnóstico temprano de esta enfermedad permitirá tratar los primeros síntomas y lesiones ambulatoriamente evitando que las mismas avancen y sea necesaria la intervención quirúrgica o la aplicación de tratamientos invasivos como las quimioterapias. El porcentaje de pacientes con cáncer de Pene ha venido aumentando con el pasar de los años a causa de que la gran mayoría de los afectados son renuentes a realizarse las pruebas o chequeos de rutina para detectar dicha patología ante la aparición de los primeros síntomas. Por norma general, el tratamiento de este tipo de Cáncer se realiza de manera escalonada, cuyas decisiones obedecen al tipo y grado de patología, a la sintomatología asociada y a la progresión de la misma. El enfoque general del presente trabajo es dar a conocer la importancia que tiene el Diagnóstico oportuno de este tipo de Cáncer. Los resultados se obtuvieron mediante el desarrollo de una investigación de tipo bibliográfica, delimitada a una metodología de revisión, que permite concluir que el manejo del Cáncer de Pene puede ir desde un tratamiento simple en su grado más bajo a un tratamiento quirúrgico, por eso la importancia de un diagnóstico oportuno que puede marcar la diferencia entre la vida y la muerte de quien la padece.

Palabras clave: Patología, Condiciones y Factores de Riesgo, Diagnóstico a tiempo, Cáncer de Pene.

\section{ABSTRACT}

Penile cancer is a disease that affects the body's cells, which multiply in a disorderly manner in the area of the male reproductive system; It is one of the less frequent pathologies in the field of urology. The early diagnosis of this disease will allow treating the first symptoms and lesions outpatiently, preventing them from advancing and requiring surgical intervention or the application of invasive treatments such as chemotherapies. The percentage of patients with penile cancer has been increasing over the years because the vast majority of those affected are reluctant to perform routine tests or check-ups to detect this pathology before the appearance of the first symptoms. As a general rule, the treatment of this type of Cancer is carried out in a staggered manner, whose decisions are due to the type and degree of pathology, the associated symptomatology and the progression of the same. The general approach of the present work is to make known the importance of the opportune Diagnosis of this type of Cancer. The results were obtained through the development of a bibliographic research, delimited to a review methodology, which allows us to conclude that the management of penile cancer can range from a simple treatment in its lowest grade to a surgical treatment, so the importance of a timely diagnosis that can make the difference between the life and death of the sufferer.

Keywords: Pathology, Conditions and Risk Factors, Timely Diagnosis, Penis Cancer.

\section{RESUMO}

O câncer peniano é uma doença que afeta as células do corpo, que se multiplicam de maneira desordenada na área do sistema reprodutor masculino; É uma das patologias menos frequentes no campo da urologia. O diagnóstico precoce desta doença permitirá tratar os primeiros sintomas e lesões ambulatorialmente, impedindo-os de avançar e exigir intervenção cirúrgica ou a aplicação de tratamentos invasivos, como quimioterapias. A porcentagem de pacientes com câncer de pênis tem aumentado ao longo dos anos, porque a grande maioria das pessoas afetadas reluta em realizar testes ou exames de rotina para detectar essa patologia antes do aparecimento dos primeiros sintomas. Como regra geral, o tratamento desse tipo de câncer é realizado de maneira escalonada, cujas decisões se devem ao tipo e grau de patologia, à sintomatologia associada e à progressão do mesmo. A abordagem geral do presente trabalho é divulgar a importância do diagnóstico oportuno desse tipo de câncer. Os resultados foram obtidos através do desenvolvimento de uma pesquisa bibliográfica, delimitada por uma metodologia de revisão, que permite concluir que o manejo do câncer de pênis pode variar de um tratamento simples em seu mais baixo grau a um tratamento cirúrgico, de modo que a importância de diagnóstico que pode fazer a diferença entre a vida e a morte do sofredor.

Palavras-chave: Patologia, condições e fatores de risco, diagnóstico oportuno, câncer de pênis. 


\section{Introducción}

El cáncer de pene, es una patología relativamente poco frecuente. La máxima incidencia se encuentra en la sexta y séptima década de la vida. La etiología de esta enfermedad es poco clara. Existen ciertas condiciones asociadas que han sido implicadas como factores causales, se incluyen falta de circuncisión, procesos inflamatorios crónicos y la exposición al virus del papiloma humano.

Considerado como un tumor raro, el cáncer de pene es el gran olvidado de la oncología masculina. Según estadísticas publicadas por Grande (2017):

Este tipo de cáncer sólo supone el 1\% de todos los tumores que afectan a los hombres en España, detectándose menos de 300 casos al año. Una cifra que, sin embargo, aumenta de manera exponencial en países subdesarrollados de África, Latinoamérica y Asia, en donde su incidencia puede alcanzar hasta el $40 \%$ de todos los tumores diagnosticados en varones.

Sánchez et al. (2016) coinciden con el criterio generalizado del gremio de especialistas al indicar que "el cáncer de pene se presenta en hombres entre los 50 y 70 años de edad, representando solamente el 2-5\% de los tumores urogenitales masculinos; sin embargo, su incidencia es variable en algunos países".

En estudio publicado por Ryan (2017) se indica que:

En su mayoría los carcinomas epidermoides se originan en el glande, en el surco coronal o debajo del prepucio. Por lo general, comienzan como una lesión eritematosa pequeña, que puede estar confinada a la piel durante mucho tiempo. Estos carcinomas pueden ser fungosos y exofíticos, o ulcerativos e infiltrantes. Este último tipo produce metástasis con más frecuencia, general- mente en los ganglios linfáticos inguinofemorales superficiales y profundos, y en los ganglios pelvianos. Las metástasis en sitios distantes (pulmones, hígado, hueso, cerebro) son raras hasta las etapas avanzadas de la enfermedad.

El Instituto Nacional del Cáncer ( $\mathrm{NCl}$, por sus siglas en inglés) destaca los siguientes métodos para detectar y diagnosticar el cáncer de pene:

- Examen físico y antecedentes: examen del cuerpo para revisar el estado general de salud e identificar cualquier signo de enfermedad en el pene, como masas o cualquier otra cosa que parezca anormal. También se toman datos sobre los hábitos de salud, los antecedentes de enfermedades y los tratamientos anteriores.

- Biopsia: extracción de células o tejidos para que un patólogo los observe al microscopio y determine si hay signos de cáncer. La muestra de tejido se extrae mediante uno de los siguientes procedimientos.

- Biopsia por incisión: extracción de una parte de una masa o de una muestra de tejido que no tiene aspecto normal.

- Biopsia por escisión: extracción completa de una masa o área de tejido que no tiene aspecto normal. $(\mathrm{NCl}, 2019)$

Si se sospecha un cáncer, es necesario hacer una biopsia; si es posible, debe tomarse una muestra de los tejidos que están por debajo de la lesión. Si en la biopsia se encuentran células cancerosas, a éstas se les asignará un grado. Esto ayuda a los médicos a predecir qué tan rápido es probable que el cáncer crezca y se propague. "A las células cancerosas se les asigna un grado en base a qué tan parecidas son a las células normales. Se usan los grados 1 , 2 , y 3. Los cánceres con un grado más alto (3) tienden a crecer más rápido". (Sociedad Norteamericana Contra el Cancer [ACS], 2018). 
El propósito fundamental de la presente investigación es plasmar todo lo relacionado con el Diagnostico del Cáncer de Pene, incluyendo conceptos, síntomas y tratamientos a los efectos de una mejor comprensión del objeto general.

\section{Materiales y Métodos}

El presente trabajo de investigación, enmarcado en una metodología de revisión, está orientado a la construcción de un material bibliográfico actualizado, enfocado en dar a conocer los criterios vigentes de los distintos Procedimientos clínicos de Diagnóstico del Cáncer de Pene.

Entre las bases de datos consultadas destacan: SciELO, NCBI, Science Direct, Pubmed, y demás fuentes de información, tales como los portales web de la Sociedad Norteamericana contra el Cáncer, el Instituto Nacional del Cáncer de los EUA, Mayo Clinic, entre otros. Como términos de búsqueda se utilizaron las expresiones "Cáncer de Pene", "Diagnóstico del Cáncer de Pene", y se aplicaron criterios de selección tales como: idioma español; publicación entre 2010 y 2020 (ambos inclusive), salvo algunas excepciones; acceso completo y abierto; en el área de salud y medicina; estudios referidos a humanos; tipo de bibliografía, artículos científicos, manuales médicos, guías clínicas, ensayos clínicos, estudios o reportes de casos, tesis de grado, posgrado y doctorado, noticias científicas, boletines y/o folletos de instituciones oficiales o privadas de excelente trayectoria en el área de la salud, medicina o científico académica, y demás, monografías y otros documentos que, a criterio propio, mostraran información de interés en base a la observación de la evidencia científica referida en sus contenidos. Este proceso arrojó resultados que en promedio oscilaron entre 7 y 69 enlaces a fuentes de información bibliográficas.

De tal manera fueron adelantadas otras búsquedas menores sin considerar la apli- cación de cualquier otro criterio de descarte, ya que se requirió encontrar información complementaria que independientemente de su origen o época de publicación, es considerada igualmente importante ajustada y de relevancia para este tema. Es a partir de entonces que se procedió con la lectura crítica y análisis interpretativo de un pilar de información recopilado a lo largo de la investigación, que también fue adoptada como evidencia. Resultando todo este proceso en la selección de los elementos más sustanciales y significativos de las diferentes fuentes bibliográficas que fundamentan el razonamiento aquí mostrado.

\section{Resultados}

El cáncer de pene es una enfermedad por la que se forman células malignas en los tejidos del pene. Los signos del cáncer de pene incluyen úlceras, secreción y hemorragia. (NCl, 2019).

Medina et al. (2010) definen al cancer de pene como:

El proceso proliferativo y desordenado de las células epiteliales escamosas del pene. Para fines prácticos, los tumores del pene se desarrollan frecuentemente a partir de la piel que lo recubre y del tejido que recubre al glande. El cáncer de pene, es una patología relativamente poco frecuente. La máxima incidencia se encuentra en la sexta y séptima década de la vida. La etiología de esta enfermedad es poco clara. Existen ciertas condiciones asociadas que han sido implicadas como factores causales, se incluyen falta de circuncisión, procesos inflamatorios crónicos y la exposición al virus del papiloma humano.

Esta patología se presenta en hombres de aproximadamente 50 años de edad y representa del 2 al $5 \%$ de los tumores urogenitales masculinos, pero su incidencia es muy variable, en algunos países puede llegar al $10 \%$ de las enfermedades malignas mas- 
culinas. Pedroso et al. (2011).

De acuerdo a lo indicado por Pizzocaro et al. (2010):

En los países occidentales, el cáncer de pene primario es infrecuente, con una incidencia inferior a 1 por 100.000 varones en Europa y los Estados Unidos. Por el contrario, en el mundo no occidental, la incidencia de cáncer de pene es mucho mayor y puede suponer el 10\%-20\% de las neoplasias malignas en los varones, con una incidencia ajustada por la edad que oscila entre 0,7-3 por 100.000 en la India y 8,3 por 100.000 varones en Brasil, e incluso mayor en Uganda, donde es el cáncer diagnosticado con mayor frecuencia. Los factores de riesgo más importantes comprenden hábitos socioculturales y prácticas higiénicas y religiosas. El carcinoma de pene es raro en las comunidades que practican la circuncisión en los recién nacidos o antes de la pubertad (judíos, musulmanes e lbos de Nigeria). La circuncisión precoz reduce el riesgo de cáncer de pene en 3-5 veces. La circuncisión en la edad adulta no protege frente al cáncer de pene.

El NCl (2019) establece la siguiente clasificación celular del cáncer de pene:

Casi todos los carcinomas de pene surgen en las células escamosas e incluyen los siguientes subtipos: Carcinoma verrugoso, Carcinoma condilomatoso (verruciforme), Carcinoma basaloide. Aunque el carcinoma condilomatoso y el carcinoma basaloide son subtipos menos frecuentes, exhiben una relación más fuerte con los virus del papiloma humano (VPH), en especial con el VPH, que el carcinoma de células escamosas típico o el carcinoma verrugoso de pene. Además, también se observan carcinomas neuroendocrinos.

\section{Factores de Riesgo}

El cáncer de pene es poco frecuente. Su causa exacta se desconoce. Sin embargo, la Enciclopedia Médica A.D.A.M. (2018) establece ciertos factores de riesgo que incluyen:

- Los hombres no circuncidados que no mantienen limpia el área por debajo del prepucio. Esto lleva a la acumulación de esmegma, una sustancia maloliente con apariencia de queso que se encuentra por debajo del prepucio,

- Antecedentes de verrugas genitales o el virus del papiloma humano (VPH).

- Tabaquismo.

- Lesión del pene.

En concordancia con esto, Mayo Clinic (2018) enumera los siguientes factores que pueden aumentar los riesgos de padecer cáncer testicular:

- Testículos no descendidos (criptorquidia). Los testículos se forman en la zona abdominal durante el desarrollo fetal, y generalmente descienden al escroto antes del nacimiento. Los hombres que tienen un testículo que nunca descendió se enfrentan a un riesgo mayor de tener cáncer testicular que los hombres cuyos testículos descendieron normalmente.

- Desarrollo testicular anormal. Las afecciones que hacen que los testículos se desarrollen en forma anormal, como el síndrome de Klinefelter, pueden aumentar el riesgo de padecer cáncer testicular.

- Antecedentes familiares. Si hay hombres en su familia que han tenido cáncer testicular, puede correr mayor riesgo.

\section{Diagnóstico}

Desafortunadamente, el cáncer de pene a menudo no se diagnostica precozmente, porque es poco común. Los médicos generales, e incluso los urólogos, se encuentran con casos de cáncer de pene sólo dos o tres veces en toda su vida profesional. Además, los pacientes a menudo no desean Ilamar 
la atención sobre sus genitales y pueden temer a los procedimientos quirúrgicos o a los tratamientos que se realizan en el pene. La presencia de una lesión en el pene es el primer indicio clínico de esta entidad. La mayoría de los pacientes han tenido estas lesiones por más de un año, tratándose con medicamentos sintomáticos y sin mejoría.

Para establecer con precisión el diagnóstico del cáncer de pene, se debe examinar la lesión primaria, los ganglios linfáticos regionales y las metástasis a distancia y someterlos a biopsia. De la lesión primaria debe determinarse el tipo histológico, diámetro de la lesión y áreas sospechosas, localización en el pene, número de lesiones, morfología y relación con otras estructuras (submucosas, cuerpo esponjoso o cavernoso, uretra), color y límites.

En este orden de ideas Medina et al. (2010) Refieren que:

El diagnóstico histológico es esencial antes de iniciar el tratamiento; el propósito no sólo es confirmar la entidad patológica sino determinar el grado tumoral. Los ganglios regionales se pueden evaluar clínicamente mediante tomografía axial computadorizada, linfografía y en años recientes azul de isosulfán o sulfuro de tecnecio-99 coloidal. La profundidad del tumor a nivel peneano es difícil de establecer por métodos de imagen, pero puede valorarse con resonancia magnética nuclear y ultrasonidos. Algunos estudios recomiendan realizar biopsia del ganglio centinela, sin embargo es controversial debido al alto porcentaje de falsos negativos (9\% a 50\%). Sin embargo, se ha ganado más experiencia con la biopsia de ganglio centinela por lo que puede ser una opción en el manejo actual. La presencia de metástasis a distancia se valora con radiografía de tórax, tomografía axial computarizada, resonancia magnética nuclear, gammagrama óseo y determinaciones bioquímicas de enzimas hepáticas y calcio sérico.

\section{Diagnóstico Diferencial}

Es probable que la mayoría de estas lesiones estén relacionadas con infecciones bacterianas, o que incluso sean reacciones alérgicas, en cuyo caso, todas responderán rápidamente a ungüentos y cremas antibacterianas o antimicóticos. Pero los crecimientos de áreas que vuelven a presentarse o que no sanan deben considerarse malignos hasta que se pruebe lo contrario. Una evaluación adecuada incluye una biopsia.

En este sentido en el sitio web especializado en cáncer Oncolink (2020) refieren que entre las pruebas para determinar el estadio del cáncer se pueden destacar las siguientes:

- Examen físico: Este es un examen general para examinar su cuerpo y hablar sobre sus problemas de salud anteriores.

- Biopsia: Una biopsia toma células del cáncer, o una parte del cáncer, para ver qué tipo de cáncer es y cómo se ve al microscopio. Un médico llamado patólogo examina la muestra en un laboratorio. Los tipos de biopsia para diagnosticar el cáncer de pene incluyen biopsia con aguja fina, biopsia incisional, biopsia excisional, biopsia de ganglio linfático centinela y/o disección de ganglio linfático.

- Imágenes: Las pruebas de radiología pueden mirar dentro de su cuerpo para observar el cáncer y determinar si se ha propagado. Estos exámenes pueden incluir los siguientes: Tomografía computarizada (TC), Resonancia magnética (RM), Tomografía por emisión de positrones (PET) y Ecografía.

Cuando se diagnostica a tiempo el cáncer del pene es sumamente curable. Debido a la rareza de este cáncer, los ensayos clínicos específicamente para cáncer del pene son poco frecuentes. Los pacientes con cáncer en niveles avanzados pueden ser candidatos para ensayos clínicos donde se evalúan fármacos nuevos, productos bioló-

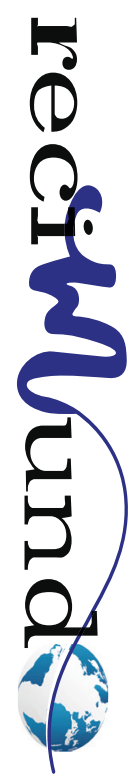


gicos o técnicas quirúrgicas para mejorar el control local y las metástasis distantes. La selección del tratamiento depende del tamaño, ubicación, invasión y estadio del tumor.

\section{Conclusiones}

El cáncer de pene es una enfermedad que se presenta en hombres de aproximadamente 50 años de edad y representa del 2 al 5\% de los tumores urogenitales masculinos, pero su incidencia es muy variable, en algunos países puede llegar al $10 \%$ de las enfermedades malignas masculinas.

Dentro de los factores predisponentes existe una relación directa entre la aparición de esta enfermedad con factores irritativos y carcinogeneticos que existen en el esmegma. La fimosis es un factor condicionante ya que impide una adecuada higiene, con aumento del tiempo de exposición al esmegma. El cáncer de pene se presenta como una lesión visible a nivel del glande siempre que el prepucio pueda rebatirse. De no ser así la lesión puede palparse a través de él como una zona irregular, endurecida. En ocasiones ulcera y perfora el prepucio, exteriorizándose hacia el exterior.

La tardanza en la consulta y, por tanto lo avanzado del cuadro en el momento de su diagnóstico, han determinado que exista una gran dificultad en su pesquisa precoz y tratamiento exitoso.

Es destacable que el manejo de esta enfermedad se debe enfocar a la educación para la detección precoz con el objetivo de intentar obtener un control más efectivo de la misma.

\section{Bibliografía}

Medina, E., Ibáñez, A., Martínez, R., Barra, R., Quezada, I., Pérez, J., \& Pérez, R. (11 de 2010). Cáncer de pene. Revisión de las características clínico-patológicas. Gaceta Mexicana de Oncología, 09(06), 282-296. Recuperado el 02 de 02 de 2020, de https://www.elsevier.es/es-revista-gaceta-mexicana-oncologia-305-articulo-can- cer-pene-revision-caracteristicas-clinico-patologicas-X1665920110004344

Pizzocaro, G., Algaba, F., Solsona, E., Tana, S., Van Der Poel, H., Watkin, N., \& Horenblas, S. (04 de 2010). European Association of Urology. Recuperado el 02 de 02 de 2020, de https://uroweb. org/wp-content/uploads/05-GUIA-CLINICA-CANCER-PENE.pdf

Sánchez, A., Martínez, G., Torres, J., \& Bernal, J. (10 de 03 de 2016). Penectomía parcial como tratamiento para el cáncer. Revista Mexicana de Urologia, 182-188. Recuperado el 03 de 02 de 2020, de https://core.ac.uk/download/pdf/82511089.pdf

Grande, E. (08 de 11 de 2017). El cáncer de pene, un gran desconocido de la oncología masculina. acta sanitaria. Recuperado el 03 de 02 de 2020, de https://www.actasanitaria.com/el-cancer-de-pene-un-gran-desconocido-de-la-oncologia-masculina/

Instituto Nacional del cancer (NIH). (16 de 04 de 2019). Instituto Nacional del cancer (NIH). Recuperado el 30 de 01 de 2020, de Instituto Nacional del cancer (NIH): https://www.cancer.gov/espanol/ tipos/pene/paciente/tratamiento-pene-pdq

Mark, J. R. (11 de 2017). Manual MSD. Recuperado el 02 de 02 de 2020, de https://www.msdmanuals. com/es-ve/professional/trastornos-urogenitales/ c\%C3\%A1ncer-urogenital/c\%C3\%A1ncer-de-pene

Mayo Clinic. (26 de 04 de 2018). Mayo Clinic. Recuperado el 30 de 01 de 2020, de https://www. mayoclinic.org/es-es/diseases-conditions/testicular-cancer-care/symptoms-causes/syc-20352986

Medlineplus. (13 de 02 de 2020). Medlineplus. Recuperado el 02 de 02 de 2020, de https://medlineplus.gov/spanish/ency/article/001276.htm

Oncolink. (15 de 01 de 2020). oncolink. Recuperado el 03 de 02 de 2020, de https://es.oncolink. org/tipos-de-cancer/cancer-de-pene/treatments/ procedimientos-quirurgicos-penectomia-para-tratar-el-cancer-de-pene

Pedroso, J., Cervantes, J., Gomez, L., \& Hernandez, R. (05 de 2011). Cáncer de pene: presentación de un caso. Revista Archivo Médico de Camagüey, 15(3).

Sociedad Americana Contra el Cancer (ACS). (25 de 06 de 2018). Sociedad Americana Contra el Cancer (ACS). Recuperado el 30 de 01 de 2020, de Sociedad Americana Contra el Cancer (ACS): https://www.cancer.org/es/cancer/cancer-de-pene/ si-usted-tiene-cancer-de-pene.html 


\section{CITAR ESTE ARTICULO:}

Garcés Erazo, K., Quisilema Ron, V., Pacheco Romero, K., \& Bungacho Moreno, D. (2020). Diagnóstico en cáncer de pene. RECIMUNDO, 4(1(Esp)), 114121. doi:10.26820/recimundo/4.(1).esp.marzo.2020.114-121

\section{cc}

(1) (2) (2)

BY NC SA

RECONOCIMIENTO-NOCOMERCIAL-COMPARTIRIGUAL CC BY-NC-SA

ESTA LICENCIA PERMITE A OTROS ENTREMEZCLAR, AJUSTARY CONSTRUIR A PARTIR DE SU OBRA CON FINES NO COMERCIALES, SIEMPRE YONSTRUIR A PARTIR DE SU OBRA CON FNES NO COMERCIALES, SIEMPRE
Y CUANDO LE RECONOZCAN LA AUTORÍA Y SUS NUEVAS CREACIONES ESTÉN BAJO UNA LICENCIA CON LOS MISMOS TÉRMINOS. 